- Row 1: A combined, flat PDF containing any Supplementary Text, Discussion, Notes, Additional Supplementary Figures, Supplementary Protocols, simple tables, and all associated legends. Only one such file is permitted.

- Row 2: Nature Research's Reporting Summary; if previously requested by the editor, please provide an updated Summary, fully completed, without any markups or comments. (Reporting Summaries are not required for all manuscripts.)

\begin{tabular}{|l|l|l|l|}
\hline Item & Present? & $\begin{array}{l}\text { Filename } \\
\text { This should be the } \\
\text { name the file is saved } \\
\text { as when it is } \\
\text { uploaded to our } \\
\text { system, and should } \\
\text { include the file } \\
\text { extension. The } \\
\text { extension must be } \\
\text { sdf }\end{array}$ & $\begin{array}{l}\text { A brief, numerical description of file } \\
\text { contents. } \\
\text { i.e.: Supplementary Figures 1-4, Supplementary } \\
\text { Discussion, and Supplementary Tables 1-4. }\end{array}$ \\
\hline $\begin{array}{l}\text { Supplementary } \\
\text { Information }\end{array}$ & No & $\begin{array}{l}\text { nr-reporting- } \\
\text { summary_NME } \\
\text { D- } \\
\text { L102212B.pdf }\end{array}$ & \\
\hline Reporting Summary & Yes & &
\end{tabular}

Editor summary: Increased perivascular fibroblast activity and vascular remodeling occurs early in sporadic ALS pathogenesis and can predict patient survival time

Reviewer recognition statement: Nature Medicine thanks Robert Baloh and the other, anonymous, reviewers for their contribution to the peer review of this work.

Title: Altered perivascular fibroblast activity precedes ALS disease onset.

Authors: Anna Månberg ${ }^{1 \#}$, Nathan Skene ${ }^{2,3,4 \#}$, Folkert Sanders ${ }^{5}$, Marta Trusohamn ${ }^{2}$, Julia Remnestål ${ }^{1}$, 
Joke De Vocht ${ }^{9}$, Koen Poesen ${ }^{8,10}$, Mathias Uhlén ${ }^{11,12}$, Jasper Anink ${ }^{13}$, Caroline Mijnsbergen ${ }^{13}$, Hermieneke Vergunst-Bosch ${ }^{14}$, Annemarie Hübers ${ }^{15,16}$, Ulf Kläppe ${ }^{17}$, Elena Rodriguez-Vieitez ${ }^{18}$ Jonathan D. Gilthorpe ${ }^{7}$, Eva Hedlund ${ }^{12}$, Robert A. Harris ${ }^{5}$, Eleonora Aronica ${ }^{13}$, Philip Van Damme ${ }^{9}$, Albert Ludolph ${ }^{15,19}$, Jan Veldink ${ }^{14}$, Caroline Ingre ${ }^{17}$, Peter Nilsson ${ }^{1}$, Sebastian A. Lewandowski ${ }^{1,5^{*}}$

\section{Affiliations and research teams:}

${ }^{1}$ Div. of Affinity Proteomics, Dept. of Protein Science, KTH Royal Institute of Technology, SciLifeLab, Stockholm, Sweden

${ }^{2}$ Dept. of Medical Biochemistry and Biophysics, Karolinska Institute, Stockholm, Sweden

$53{ }^{3}$ Division of Neuroscience, Dept. of Brain Sciences, Imperial College London, London, United Kingdom

$54{ }^{4}$ United Kingdom Dementia Research Institute, London, United Kingdom

$55{ }^{5}$ Karolinska Institute, Dept. of Clinical Neuroscience, Centre for Molecular Medicine, Karolinska

56 Hospital, Stockholm, Sweden

$57 \quad{ }^{6}$ Dept. of Laboratory Medicine, Karolinska Institute, Stockholm, Sweden

$58{ }^{7}$ Umeå University, Dept. of Integrative Medical Biology, Umeå, Sweden

$59{ }^{8}$ Laboratory for Neurobiomarker Research, Dept. of Neurology, Leuven Brain Institute, KU Leuven

60 (University of Leuven), Leuven, Belgium

$61{ }^{9}$ KU Leuven, Neurology Dept. and Center for Brain \& Disease Research, VIB, Leuven, Belgium

$62{ }^{10}$ Laboratory Medicine, UZ Leuven (University Hospital Leuven), Leuven, Belgium

$63{ }^{11}$ Div. Systems Biology, Dept. Protein Science, KTH Royal Institute of Technology, SciLifeLab,

64 Stockholm, Sweden

$65{ }^{12}$ Dept. Neuroscience, Karolinska Institute, Stockholm, Sweden

$66{ }^{13}$ Amsterdam UMC, University of Amsterdam, Dept. of (Neuro)Pathology, Amsterdam Neuroscience, 67 Amsterdam, The Netherlands

$68{ }^{14}$ UMC Utrecht Brain Center, University Medical Center Utrecht, Dept. of Neurology, Utrecht 69 University, Utrecht, The Netherlands

${ }^{15}$ University of Ulm, Neurology Clinic, Ulm, Germany

${ }^{16}$ Current affiliation: Neurology Division, Geneva University Hospital.

${ }^{17}$ Dept. of Clinical Neuroscience, Karolinska Institute, Stockholm, Sweden. Dept of Neurology at Karolinska University Hospital. Institutet, Stockholm, Sweden

${ }^{19}$ Deutsches Zentrum für Neurodegenerative Erkrankungen (DZNE), Ulm

\# These authors contributed equally.

* Corresponding author: sebastian.lewandowski@ki.se 


\section{Abstract:}

81 Apart from the well-defined factors in neuronal cells ${ }^{1}$, only few reports consider that variability of

82 sporadic ALS progression can depend on the less-defined contributions from glia ${ }^{2,3}$ and blood vessels ${ }^{4}$.

83 In this study we use an expression weighted cell-type enrichment method to infer cell activity in spinal

84 cord samples from sporadic ALS patients and mouse models of this disease. Here we report that

85 sporadic ALS patients present cell activity patterns consistent with two mouse models in which

86 enrichments of vascular cell genes preceded the microglial response. Notably, during the

87 presymptomatic stage, perivascular fibroblast cells showed the strongest gene enrichments and their

88 marker proteins SPP1 and COL6A1 accumulated in enlarged perivascular spaces in sporadic ALS

89 patients. Moreover, in plasma of 574 ALS patients from four independent cohorts, increased levels of

90 SPP1 at disease diagnosis repeatedly predicted shorter survival with stronger effect than the

91 established risk factors of bulbar onset or neurofilament levels in cerebrospinal fluid. We propose that

92 the activity of the recently-discovered perivascular fibroblast can predict ALS patient survival and

93 provide a novel conceptual framework to re-evaluate definitions of ALS etiology. 


\section{Introduction}

Although the defining clinical features of amyotrophic laterals sclerosis (ALS) focus on the common degeneration of upper and lower motor neurons, substantial variability is reported for patient's age at disease onset, contribution of non-motor systems and survival duration ${ }^{5}$. This variability considerably complicates accurate prognosis of life expectancy and interpretations of clinical trial outcomes. Lack of reliable early diagnostics means that treatments are given only in advanced stages; and further highlights that studies aiming to understand early sporadic disease mechanisms are intrinsically challenging and incomplete ${ }^{6}$.

From the first description of familial ALS forms ${ }^{7}$ which affect $5-10 \%$ of patients, there have been remarkable advances in discovery of novel gene variants. These variants provided insight into dysfunction of neuron-centric pathological mechanisms including protein misfolding ${ }^{7}$, RNA maturation $^{8}$ and axonal transport ${ }^{9}$. However, increasing evidence indicates that the dynamics of neurodegeneration in both familial and sporadic ALS is also influenced by other cell type functions that include glia response ${ }^{2,3}$, oligodendrocyte metabolism ${ }^{10}$ and integrity of blood vessels ${ }^{4,11}$. These observations challenged the neuron-centric theory and initiated redefinition of our understanding of disease variability. Recent efforts to comprehend cellular complexity of the nervous system using single cell RNA sequencing provided detailed maps of novel cell types ${ }^{12-14}$ but also add another level of challenge to interpret individual cell-type input during multiple stages of disease. Unless we improve our understanding of contributions from non-neuronal cells and mechanisms preceding disease onset, the clinical variability of the progression dynamics will continue to confound design and evaluation of ALS treatments. We therefore aimed to decode the temporal activity for gene expression within ten major central nervous system cell types in post-mortem transcriptomes of patients with sporadic ALS and in a presymptomatic timeline of the transgenic SOD $1^{G 93 A}$ and TARDBP $P^{0331 K / 0331 K}$ mouse models.

\section{Cell activity timeline in ALS}

We used single-cell central nervous system transcriptomes ${ }^{12,13}$ and an expression weighted cell-type enrichment (EWCE) ${ }^{15}$ method to infer the cell activity in bulk tissue. This form of inferred activity is represented as a z-score which measures the degree of transcript increase and specificity within a given cell category derived from single-cell sequencing. In our analysis we included transcriptomes from spinal cords of sporadic ALS patients $(n=12)$ and two transgenic mouse models: $\operatorname{SOD}^{G 93 A}(n=3)$ and TARDBP $P^{0331 K / 0331 K}$ ( $\mathrm{n} \geq 8$ ) (Fig. $1 \mathrm{a}, \mathrm{b}$ Extended Data Fig. 1, 2). The results from sporadic ALS patient tissues revealed increased enrichments of microglia and astrocyte-specific genes together with a marked reduction of excitatory neuron and interneuron transcripts likely reflecting neurodegeneration (Fig. 1 b). Notably we observed increased activity of vascular cell-specific genes in sporadic ALS patients with the highest enrichments for perivascular fibroblast cells (Fig. $1 \mathrm{~b}$ ). To complement the endpoint patient data and to explore presymptomatic patterns of cell activity we analyzed gene enrichments for the same ten cell types in $S O D 1^{G 93 A}$ and TARDBP $P^{Q 331 K / Q 331 K}$ mice. We analyzed SOD $1^{G 93 A}$ 
131 mice at ages of 4, 6 (asymptomatic); 8 (neuromuscular junction decoupling ${ }^{16}$ ); 10 (pre-onset); 14

132 (onset of neuroinflammation ${ }^{17}$ ); 16 (peak body weight - clinical onset with loss of motor neuron

133 count $^{18}$ ) and 18 weeks (symptomatic stage). TARDBP $0331 K / 0331 K$ mice were analyzed at 5 month (no MN

134 loss or NMJ denervation with partial behavior phenotype ${ }^{19}$ ) and 20 month (with behavioral phenotype)

135 timepoints. We observed a remarkable consistency in cell type activity between end-stage sporadic

136 ALS patients and symptomatic SOD1 $1^{\text {G93A }}$ or TARDBP ${ }^{0331 K / 0331 K}$ mice with similar increased expression of

137 microglia and astrocyte associated genes as well as a reduction of neuron and interneuron transcripts

138 (Fig. 1b, Extended Data Fig. 1). Our observations in SOD $1^{G 93 A}$ mice were consistent with previously

139 published FACS sorting ${ }^{20}$ and RNA pulldown ${ }^{21}$ approaches, which also aimed to identify cell type-

140 specific transcripts. For instance, we identified Trem2, Ctss with Cd86 as well as Sprr1a with KIk6 as

141 respective microglia and oligodendrocyte-specific regulated genes, similarly to the previous reports

142 from in SOD $1^{G 93 A 20}$ and SOD $1^{\text {G37R } 21}$ models (Extended Data Fig. 1). In comparison with those reports,

143 our dataset not only includes novel cell types, but also resolves with a more refined timescale to

144 demonstrate that microglia and astrocytes become induced at pre-onset week 14, while neuron and

145 interneuron genes already show decreased activity in presymptomatic 8 week-old mice.

146 Remarkably, in the $S O D 1^{G 93 A}$ model the response of vascular cells including pericyte- (4-6

147 weeks), smooth muscle- (8 weeks) and endothelial cell-specific genes (10 weeks) largely occurred

148 before the microglial response (week 14-18) and showed strongest enrichment for perivascular

149 fibroblast-specific genes (4, 8, 14, 16 and 18 weeks) (Fig. 1b and Extended Data Fig. 2a). A similar

150 increase of perivascular fibroblast-specific genes was also evident and preceded microglial

151 transcriptional activity in 5 month-old TARDBP $P^{0331 K / 0331 K}$ mice (Fig. 1 b). The timing and degree of

152 perivascular fibroblast-specific gene expression could imply that the extent of their activity is similar to

153 microglia, but they become induced at much earlier stages of disease. We therefore focused our

154 analysis on the perivascular fibroblast-specific expression patterns and defined several activated genes

155 in human sporadic ALS patients as well as in SOD $1^{\text {G93A }}$ and TARDBP $P^{0331 K / 0331 K}$ mice (Fig. 1c, d, e). The

156 timing of expression for perivascular fibroblast-specific genes in $S O D 1^{G 93 A}$ mice (Fig. 1e) implies that

157 one group becomes active during the presymptomatic stage of 4-8 weeks, while another group

158 become active together with the microglial response initiated at week 14 . We found similar patterns of

159 early and late responses of perivascular fibroblast-specific genes in TARDBP $P^{0331 K / 0331 K}$ mice (Fig. 1 e).

160 Perivascular fibroblasts were recently identified as a unique nervous system cell type by other

161 groups using single-cell RNA sequencing efforts ${ }^{13,22}$ (Fig. 1f) and are also referred to as vascular

162 leptomeningeal cells ${ }^{13,14}$. These cells were previously reported to locate within the perivascular

163 Virchow-Robin space between the mural cells and astrocyte end-feet ${ }^{22}$ (Fig. 1g). Our transmission

164 electron microscopy observations further specify the location of perivascular fibroblasts between the

165 astrocyte and mural cell basement membranes (Fig. 1h). Perivascular fibroblast-specific genes (e.g.

166 Col6a1, Col1a1 and $M m p 2)^{13,22}$ imply that their main functions include composition and remodeling of

167 the basement membrane extracellular matrix ${ }^{22}$. However, as current tools are inadequate to perform 
recombinant cell-specific interventions, the precise role of these cells in the nervous system remains highly debated. To enable the exploration of our dataset we have built a web resource with cell typespecific enrichment rankings, transcript specificity and expression dynamics in sporadic ALS patients, $S O D 1^{G 93 A}$ and TARDBP $P^{0331 K / 0331 K}$ mice at http://alscellatlas.org ).

Since perivascular fibroblast cells in our data were the first to induce gene expression before ALS onset and their function is largely unknown, we focused on addressing their potential contributions to disease processes. Our computational predictions were based on RNA expression specificity in a healthy state and we needed to verify if perivascular fibroblast protein expression indeed localized within blood vessels in ALS tissues. We performed a histological screen with antibodies targeting 15 perivascular fibroblast-specific proteins. The analysis confirmed vascular location for several proteins, in particular COL6A1 and SPP1 (osteopontin) (Fig. 2a, b and Extended Data Fig. 3). Stainings of both proteins were increased in spinal cords of sporadic ALS patients as compared with age-matched non-neurological controls (Fig. 2c, Extended Data Fig. 3a). In addition, vessel-specific increase of Spp1 and Col6a1 protein expression in SOD $1^{G 93 A}$ mice spinal cords (Fig. 2d) was consistent with those observed in sporadic ALS patients. Accumulation of both proteins already occurred at an early timepoint of 8 weeks (Fig. 2e), which supports the inferred notion from the RNA transcriptomics data that perivascular fibroblasts become induced at presymptomatic stage of ALS.

Notably, staining for COL6A1, a component of the vascular basement membrane, did not outline endothelial or mural cells, but indicated enlarged perivascular spaces in ALS patients (Fig. $2 \mathrm{~b}$ and Extended Data Fig. 3a) suggesting that astrocyte and mural basement membranes become separated during ALS progression. We confirmed that separation of mural basement membrane (marked with COL4A1) and astrocyte basement membrane (marked with LAMA1) does indeed occur in spinal cord vessels in sporadic ALS patients (Extended Data Fig. 3b). A similar separation of Col4a1 and Lama1-delineated basement membranes already occurred in presymptomatic ( 8 week-old) SOD $1^{\text {G93A }}$

194 mice (Fig. 2f) at a timepoint consistent with increased transcriptional activity and protein accumulation 195 of perivascular fibroblast-specific markers. We resolved the ultrastructure of basement membrane 196 separation using transmission electron microscopy and observed that perivascular fibroblasts were 197 present within enlarged perivascular spaces in 14 week-old SOD $1^{G 93 A}$ mice (Fig. $2 \mathrm{~g}$ ). We found that 198 these perivascular spaces accumulate perivascular fibroblast-specific COL6A1 and SPP1 proteins in spinal cord vessels of sporadic ALS patients (Fig. 2h, i and Extended Data Fig. 4a). The enlargement of perivascular spaces was substantial in both end-stage ALS patients and in 14 week-old SOD $1^{G 93 A}$ mice (Fig. 2j). This form of basement membrane remodeling is likely regulated by multiple genes, as indicated by enriched gene ontologies which occurred early and were exacerbated in symptomatic stages of ALS (Extended Data Fig. 5). Taken together, these findings indicate that perivascular fibroblasts become active during presymptomatic ALS stages and can participate in remodeling of 
cerebral blood vessels by increased expression of the COL6A1 and SPP1 proteins within enlarged perivascular spaces (Fig. 2k).

\section{Prognostic value of perivascular fibroblast proteins in plasma}

209 Since perivascular spaces are responsible for clearance from cerebrospinal fluid to lymphatic ${ }^{23}$ and

210 blood $^{24}$ circulations, we anticipated that an increase of COL6A1 and SPP1 in ALS patient plasma could

211 have potential informative value for clinical disease dynamics. We performed targeted suspension

212 bead array profiling using 32 antibodies against 15 perivascular fibroblast proteins in blood plasma.

213 Initial screening revealed a that higher quartile values of COL6A1 and SPP1 proteins indicated shorter

214 survival of ALS patients in a pilot cohort from the Netherlands (189 ALS patients and 199 controls)

215 (Extended Data Fig. 7a). We have combined the Netherlands cohort with groups from Germany and

216 Belgium (a total of 452 ALS patients and 395 controls) and analyzed it as a discovery cohort with

217 internal replication between countries. We have also recruited samples from Sweden (122 ALS

218 patients and 109 controls) as a replication cohort with independent labeling of beads with antibodies.

219 Cohort summary statistics are presented in Extended Data Fig. 6.

220 SPP1 levels above empirically optimized thresholds indicated shorter survival in the discovery

221 and replication cohorts (Fig. 3a). These associations of high SPP1 and shorter survival were reproduced

222 within the individual cohorts from Netherlands and Germany but not in the smaller cohort from

223 Belgium with less complete survival data (Extended Data Fig. 8). We also performed a validation using

224 a second SPP1 antibody targeting an independent protein domain (Extended Data Fig. 7b). This

225 antibody showed similar association between high SPP1 values and short patient survival in each

226 country cohort (Extended Data Fig. 7, 8b, 9a). Plasma COL6A1 level increase also associated with

227 shorter survival in the grouped discovery cohort and within the larger groups from Netherlands

$228(\mathrm{n}=189)$ and Germany ( $n=187)$, but not in the smaller cohorts with less complete survival data from

229 Belgium ( $n=102)$ and Sweden ( $n=122)$ (Extended Data Fig. 8c, 9a).

230 Besides the comparison of patient groups defined by protein level threshold, we also

231 evaluated the prognostic risk for continuous increase of plasma SPP1 and COL6A1 in univariate and

232 multivariate Cox models. We compared their hazard ratios to the effects of bulbar onset and, when

233 available, neurofilament light (NFL) levels. Using a univariate model, the hazard ratio for continuous

234 plasma increase of SPP1 in the grouped discovery cohort was higher (HR=1.82 $p=8.96 \mathrm{e}-05)$ than the

235 risk mediated by the bulbar onset ( $H R=1.44 p=2.1 e-05)$ (Fig. 3c). This effect of univariate risk increase

236 was consistent in the Swedish replication cohort in which SPP1 increase had higher hazard ratio

$237(\mathrm{HR}=3.27 \mathrm{p}=0.0008)$ than bulbar onset $(\mathrm{HR}=1.66 \mathrm{p}=0.0378)$ or NFL increase in CSF (HR=1.61 $\mathrm{p}=0.002)$

238 (Fig. 3c). We have further confirmed these observations using the second antibody for SPP1 and

239 univariate Cox models in discovery and replication cohorts (Extended Data Fig. 9b). In addition to the

240 univariate model, we cross-examined the effects of continuous protein increase using a multivariate

241 Cox model in which SPP1 persisted to give stronger survival hazard ratios ( $H R=1.82 p=8.96 e-05)$ than 
the bulbar onset in the discovery cohort (Fig. 3c). Strikingly, in the replication cohort where matched

243 CSF samples were available, the multivariate effect of SPP1 increase in plasma had stronger indication

244 for short survival $(H R=3.56 p=0.009)$ than did the NFL increase in CSF as measured using a clinical grade

245 ELISA kit (HR=1.5 p=0.02) (Fig. 3c). The increase of SPP1 in CSF also indicated shorter survival in the

246 Swedish cohort, albeit at much higher threshold and to a lesser extent than did the plasma values

247 (Extended data Fig. 9c). The correlations between SPP1 and COL6A1 were more apparent in CSF than

248 in plasma (Extended Data Fig. 10a), which could support the proposed CNS origin of these proteins as

249 indicated by our transcriptomics and histological data. However, the relative and longitudinal increase

250 of SPP1 was more evident in ALS patient plasma rather than in CSF (Extended data Fig. 10 b, c) which

251 was concordant with a higher predictive value in plasma.

252

\section{Discussion}

254 Our findings indicate the interdependence of vascular and neuronal systems and illustrate the

255 novel, early contributions from perivascular fibroblast cells which were not previously considered in

256 interpretations of ALS etiology. We propose that inclusion of vascular cell-specific inputs will likely

257 improve prognostic accuracy at disease diagnosis and can help to re-evaluate the current view on

258 mechanisms that facilitate neurodegeneration. Although the current neuron-centric view is valid from

259 the motor phenotype standpoint, it may not be sufficient to explain the reported variety of sporadic

260 ALS phenotypes ${ }^{5,25}$ and disease dynamics ${ }^{26}$. The existing assessments of disease dynamics, which are

261 often based on neuronal axon-derived inputs (e.g. neurofilaments), general epidemiological

262 descriptions (e.g. age at onset, diagnostic delay) or physiological factors (e.g. bulbar onset, functional

263 rating scales), provide limited perspective for at least two reasons.

264 Firstly, current prognostic and causative assumptions are based on indicators of outcome (e.g.

265 neurofilament proteins and disease progression scales) which likely reflect the damage already

266 occurred, but not necessarily the early mechanisms and cell types that precede symptom onset. These

267 presymptomatic mechanisms may not be directly reflected by motor phenotypes, as indicated by our

268 mouse model observations, and are intrinsically difficult to observe in sporadic ALS patients. In

269 addition, a recent attempt to stage ALS has defined its neuronal onset in the motor cortex but, in

270 contrast to Alzheimer's and Parkinson's disease, showed no preclinical stages for neuronal cells ${ }^{27}$. Our

271 report therefore focuses on cellular activity in presymptomatic ALS mice and demonstrates that

272 induction of perivascular fibroblast transcripts, and deposition of the respective proteins largely

273 precede neuroinflammation ${ }^{17}$ and neuronal $\operatorname{loss}^{28}$.

274 Secondly, considering the interdependency of cell types in the nervous system, the net

275 outcome for ALS neurodegeneration dynamics is not only associated with, but also relies on support

276 mechanisms from multiple cell types including blood vessels. However, current molecular factors that

277 indicate survival variability in the clinics are mostly based on neuron-derived proteins and only

278 recently on concurrent microglia-derived ${ }^{29}$ proteins which reflect late symptomatic stages of disease. 
Input from these cell types likely represents only partial accuracy of early prognosis and limits the design of therapeutic or preventive clinical trials. Herein we showed that the SPP1 protein alone had a stronger indication on survival prediction than did standard physiological or neuron-based inputs when measured at first clinical visit. Moreover, the increase of SPP1 remained the strongest indicator for poor survival when compared against bulbar onset and neurofilament levels in CSF using multivariate models. survival. Even though vascular dysfunction has previously been proposed as an epidemiological risk factor in humans ${ }^{30,31}$ and is observed in animal models ${ }^{4,11}$ our results instead provide a plausible molecular explanation for these associations, a potential target for therapy as well as practical prognostic tools for the wide population of sporadic ALS patients. Although speculative at this point, vascular remodeling may be important to ALS neurodegeneration because of its reported associated effects on the blood-brain barrier function ${ }^{4}$, reduced blood flow ${ }^{32,33}$, and/or decreased cerebral glucose uptake ${ }^{34}$. The prognostic value of SPP1 levels likely represents a general vascular injury response and has been reported beyond ALS neurodegeneration in several publications showing an increase of SPP1 as a reflection of the severity of cardiovascular ${ }^{35}$, inflammatory ${ }^{36}$ and malignant ${ }^{37}$ conditions. These conditions are all likely also represented in the age-matched non-motor neuron disease control group, and could explain the low relative difference in SPP1 levels between such controls and ALS patients.

From now on, it will be important to refine the descriptions of mechanisms in presymptomatic stages of ALS. Our data resource allows exploration of early cell-specific events and also highlights the unanticipated role of perivascular fibroblast proteins in survival prognosis. We propose that detailed studies of blood vessel-derived mechanisms should become a major focus in order improve prognostic accuracy. This could help to re-evaluate ALS etiology and to inform appropriate future therapeutic approaches although validity of SPP1 as a therapeutic target remains to be addressed with interventions. Since enlarged perivascular spaces are repeatedly observed in aging brains ${ }^{38}$, in dementia $^{39}$ and in other neurological disorders ${ }^{40}$, perivascular fibroblast cell activity within those spaces could represent a common therapeutic target in cerebral injury.

\section{Materials and methods}

\section{Bioinformatics}

313 -Expression weighted cell type enrichment (EWCE)

314 Top 250 genes regulated in bulk tissue transcriptomics datasets were analyzed for overrepresentation

315 of cell-specific transcripts derived from single cell RNA-sequencing. Single cell RNA-seq data from two 
316 separate publications were used. The cortex mRNA expression data was downloaded from the

317 associated website on the Linnarsson lab home (URL, https://storage.googleapis.com/linnarsson-lab-

318 www-blobs/blobs/cortex/expression mRNA 17-Aug-2014.txt ${ }^{12}$. Duplicated gene symbols were

319 dropped. Additional single cell RNA-seq data was obtained from the Marques et al ${ }^{13}$ and downloaded

320 from the associated webpage

321 (http://www.ncbi.nlm.nih.gov/geo/download/?acc=GSE75330\&format=file\&file=GSE75330\%5FMarqu

322 es\%5Fet\%5Fal\%5Fmol\%5Fcounts2\%2Etab\%2Egz). Cells which were annotated as belonging to the cell

323 class "(none)" were dropped from both datasets. The two single cell datasets were joined using the

324 merge_two_expfiles() function from the EWCE package ${ }^{41}$. The level1class for the following cells were

325 modified: Vsmc to "Vascular Smooth Muscle Cell" ( $n=62)$; Peric to "Pericytes" ( $n=21)$; Vend1 and

326 Vend2 to "Vascular Endothelial" ( $n=137)$; astrocytes_ependymal to "Astrocytes" $(n=224)$; pyramidal SS

327 and CA1 to "Excitatory Neurons" ( $\mathrm{n}=1338$ ); NFOL*, MFOL* and MOL* to "Oligodendrocytes" ( $\mathrm{n}=4528$ );

328 OPC and COP to "Oligodendrocyte progenitors" ( $n=449)$; and PPR (Vascular and Leptomeningeal Cells)

329 to "Perivascular Fibroblasts" ( $n=76)$. The labeling of "Microglia" $(n=98)$ and "Interneuron" $(n=290)$ cell

330 categories were unchanged. Specificity data was calculated for the single cell dataset using the EWCE R

331 package (available from github.com/NathanSkene/EWCE) ${ }^{41}$.

332 A third single-cell dataset from Vanlandewijck et al. ${ }^{22}$ was used for visual reference of vascular cell

333 transcript specificity.

334

335 The mouse SOD $1^{G 93 A}$ spinal cord dataset ${ }^{42}$ was downloaded from GEO (accession GSE18597). Raw cell

336 files were obtained and loaded into $\mathrm{R}$ using the affy package ${ }^{43}$. Probe annotations and mapping to

337 HGNC symbols was done using the biomaRt R package ${ }^{44}$. All arrays were analyzed together, with a

338 separate column included in the design matrix for each age and mutants at each age. Differential

339 expression analysis was performed using the limma package ${ }^{45}$. The $T_{A R D B P^{0331 K / 0331 K} \text { mouse }^{0} \text { dataset }}{ }^{19}$

340 RNA-seq data files were obtained from GEO (accessions GSE99353 and GSE112575). Files were

341 combined, feature names were corrected manually. Differential expression analysis was performed

342 using edgeR and limma packages. The human spinal cord dataset ${ }^{46}$ was downloaded from GEO

343 (accession GSE18920). The data from enriched motor neurons was dropped and only anterior horn

344 samples were kept. Differential expression analysis was again performed using the limma package ${ }^{45}$

345 controlling for gender. EWCE analysis was performed on the top 250 up/down-regulated genes, sorted

346 based on the t-statistic, using 10,000 bootstrap replicates for each analysis.

-Gene ontology analysis

349 Human and mouse gene sets for gene ontology analysis were downloaded from geneontology.org.

350 Enrichment of the gene sets within the ALS patient and mouse SOD $1^{G 93 A}$ expression data was analyzed

351 with the mroast function from the limma R package, limiting for each platform the genes analyzed to

352 the genes where data was available on the respective platform. 
- Mouse strains, housing. The SOD1 ${ }^{G 93 A}$ (B6SJL-Tg(SOD1*G93A)1Gur/J) strain used as an ALS model were a kind gift from Prof. Stefan Marklund, Tomas Brännström and Peter Andersen at Umeå University, Sweden. B6SJL-Tg(SOD1)2Gur/J mice overexpressing wild-type SOD1 were purchased from the Jackson Laboratory. Mice were housed in individually ventilated cages in a specific pathogen free facility and given free access to food and water with 12/12hour light cycle ambient room temperature $19-23^{\circ} \mathrm{C}$ and air humidity 40-60\%. Symptomatic mice were given solid drink (\#95-23-100) from Nova SCB. Transgene-bearing mice were identified by PCR genotyping as described previously ${ }^{7}$. SOD1 copy number was determined with $\mathrm{qPCR}$ using fluorescent probes for $h S O D 1$ and $m A p o L$ and was carried as described in guidelines by the Jackson Laboratory ${ }^{47}$. Mice with more than a $0.5 \mathrm{dCt}$ differences from the $S O D 1^{G 93 A}$ reference DNA (Jackson Laboratories) were discarded from the colony.

367 All the work involving animal or human subjects or tissues has been carried out in accordance with the

368 Code of Ethics of the World Medical Association (Declaration of Helsinki) and with national legislation 369 as well as our institutional guidelines. Animal experiments were approved and performed according to 370 the guidelines of the North Stockholm Animal Ethics Committee. ALS patient plasma or CSF collection was approved by the ethics committees at UIm University (application number 20/10), UMC Utrecht (SL/nb/ 16/004075) Leuven University (ML4073) and Karolinska Institute (2018/1605).

373

Post-mortem material (Table 1.) https://figshare.com/s/4c89e57b39620d020f8d was obtained at autopsy from ALS patients at the department of (Neuro)pathology of the Amsterdam UMC, Academic Medical Center, University of Amsterdam, the Netherlands. All patients fulfilled the diagnostic criteria for ALS (El Escorial criteria) ${ }^{48}$ as reviewed independently by two neuropathologists. All patients with ALS died from respiratory failure. Control spinal cord tissue was obtained from patients who had died from a non-neurological disease. Both ALS and control patients included in the study displayed no signs of infection before death. Informed consent was obtained for the use of brain tissue and for access to medical records for research purposes and approval was obtained from the relevant local ethical committees for medical research. All autopsies were performed within 12 hours after death.

387 Paraffin-embedded tissue was sectioned at $6 \mu \mathrm{m}$ and mounted on pre-coated glass slides (StarFrost, 388 Waldemar Knittel Glasbearbeitungs GmbH, Braunschweig, Germany). Representative sections of all specimens were processed for haematoxylin and eosin and Klüver-Barrera. 
$391-$ Immunohistochemistry on spinal cord samples from sporadic ALS patients

392 For immunohistochemistry on human spinal cord samples, formalin-fixed paraffin-embedded $6 \mu \mathrm{m}$

393 thick sections were deparaffinized in xylene and rinsed in graded ethanol (100\%, 95\%, 70\%). Antigen

394 retrieval was performed in $0.01 \mathrm{M} \mathrm{HCl}$ with 0,5\% Pepsin (Sigma Aldrich, Darmstadt, Germany, \#P7012)

395 at $37^{\circ} \mathrm{C}$ for 15 minutes in a water bath followed by incubation with a given primary antibody (Collagen

396 IV, MS-747-S, Thermo Sci, 1:50; COL6A1, HPA019142, Atlas antibodies, 1:500; SPP1, AF1433, R\&D,

397 1:50; LAMA1, Sigma L9393, 1:50). Incubation with primary antibody was performed overnight at $4^{\circ} \mathrm{C}$.

398 After washing in PBS, sections were stained with a polymer based peroxidase immunohistochemistry

399 detection kit (Brightvision plus kit, ImmunoLogic, Duiven, the Netherlands) according to the

400 manufacturer's instructions. Staining was performed using Bright DAB substrate solution

401 (ImmunoLogic, Duiven, the Netherlands). Sections were dehydrated in alcohol and xylene and

402 coverslipped.

403 For double immunohistochemistry, sections were incubated with Brightvision poly-alkaline

404 phosphatase-goat-anti mouse (Immunologic, Duiven, The Netherlands) for $30 \mathrm{~min}$ at RT and washed

405 with PBS. Sections were washed with Tris- $\mathrm{HCl}$ buffer $(0.1 \mathrm{M}, \mathrm{pH} 8.2)$ to adjust the $\mathrm{pH}$. Alkaline

406 phosphatase activity was visualized with the alkaline phosphatase substrate kit III Vector Blue (SK-

4075300 , Vector laboratories Inc., CA, USA). The first primary antibody was cooked off by cooking in

$40810 \mathrm{mM}$ citrate buffer $\mathrm{pH} 6.0$ for $10 \mathrm{~min}$ at $100^{\circ} \mathrm{C}$ in a pressure cooker and subsequently incubated with

409 the second primary antibody overnight at $4^{\circ} \mathrm{C}$. The next day the sections were incubated with rabbit-

410 anti-goat (SouthernBiotech \#SBA 6164-01) for SPP1 for $15 \mathrm{~min}$ and then with Brightvision goat-anti-

411 rabbit poly HRP for $30 \mathrm{~min}$ at RT, and washed with PBS. Horseradish peroxidase was visualized with

412 filtered $5 \times 10-4 \%$ w/v AEC in in 0.05M acetate buffer $\mathrm{pH} 4,9$ and $1 \times 10-4 \% \mathrm{H}_{2} \mathrm{O}_{2}$. Sections incubated

413 without primary antibodies were blank.

414

415 Sporadic ALS was defined as without the presence of C9ORF72 hexanucleotide repeat expansion or 416 mutations analyzed by targeted NGS analysis. The NGS panel consists of TARBP, ALS2, ErbB4, NEK1, 417 MATR3, VCP, SIGMAR1, c9orf72, c19orf12, OPTN, HNRNPA1, DAO, SPG11, FUS, GRN, PNPLA6, SOD1, 418 CHCHD10, NEFH and UBQLN2.

419 For histopathological scoring, all labeled tissue sections were evaluated by two independent observers

420 blinded to clinical data for the presence or absence of various histopathological parameters and

421 specific immunoreactivity (IR) for the different markers. Hematoxylin-Eosin (HE) and Nissl stained

422 slides were used to evaluate the neuronal and glial components of the tissues. The intensity of GFAP

423 and HLA-DR (MHC-II) immunoreactive staining was evaluated using a scale of 0-3 (0: -, no; 1: +/-, weak;

$4242:+$, moderate; $3:++$, strong staining). The frequency of GFAP and HLA-DR positive cells [(1) rare; (2)

425 sparse; (3) high] was also evaluated to give information about the relative number of positive cells

426 within the spinal cord (lumbal region). As proposed before ${ }^{49,50}$, the product of these two values 
(intensity and frequency scores) was taken to give the overall score (total score; immunoreactivity score; IRS). Key scoring: Frequency: (1)<1-10\% (2) 11-50\% moderate; (3) > $50 \%$. Intensity: 0: not present; $1+$, weak; $2+$, moderate; $3+$, strong.

430 Antibodies used for scoring included: Glial fibrillary acidic protein (GFAP; polyclonal rabbit, Z0334,

431 DAKO, Glostrup, Denmark; 1:4000), neuronal nuclear protein (NeuN; mouse clone A60, MAB377

432 Merck-Millipore), major histocompatibility complex (MHC) class II antigen (HLA)-DP, DQ, DR (mouse

433 clone CR3/43; M0775, DAKO, Glostrup, Denmark; 1:400) and CD68 (mouse clone PG-M1, M0876,

434 DAKO; $1: 200)$.

-Mouse tissue immunofluorescence and confocal microscopy

Following PBS and PFA perfusion, mouse tissues were incubated with $30 \%$ sucrose solution overnight, embedded in frozen section media and snap frozen on dry ice. $16 \mu \mathrm{m}$ sections were cut on a Micron cryostat for histology staining and quantifications. The following antibodies were used for immunostainings: Podocalyxin (AF1556 R\&D; 1:250), Collagen IV (2150-1470 Serotec; 1:250), SPP1 (HPA027541, Atlas antibodies 1:250), COL6A1 (HPA019142, Atlas antibodies, 1:250) and LAMA1 (L9393, Sigma, 1:500). Secondary goat antibodies conjugated to Alexa Fluor 488, 555, 594 or 647 were purchased from Life Technologies and used at 1:500. Images were acquired using Zeiss LSM 700 confocal microscope and Zen software.

\section{Transmission electron microscopy}

447 Transmission electron microscopy was performed according to standard protocols. Briefly, mice were 448 anesthetized and perfused with PBS. Spinal cords were excised and fixed in the fixation solution buffer 449 (2\% glutaraldehyde, $0.5 \%$ paraformaldehyde, $0.1 \mathrm{M}$ cacodylate, $0.1 \mathrm{M}$ sucrose, $3 \mathrm{mM} \mathrm{CaCl}_{2}$ ) and 450 washed in $0.1 \mathrm{M}$ cacodylate buffer $(\mathrm{pH}, 7.4)$ before staining in $2 \% \mathrm{OsO} 4$ in cacodylate buffer for 1 hour at room temperature. Samples were dehydrated and en bloc staining was performed in $2 \%$ uranyl acetate in absolute ethanol for 1 hour at room temperature; samples were then taken through an Epon 812/acetone series and embedded at $60^{\circ} \mathrm{C}$ in pure Epon 812 . Thin sections of $70 \mathrm{~nm}$ thickness were made on a Leica EM UC6 Ultratome and mounted on Formvar-coated copper slot grids. Post staining was done with $2 \%$ aqueous acetate $(\mathrm{pH}, 3.5)$ and Venable and Coggleshall lead citrate. Grids were analyzed on an FEI TECNAI electron microscope.

459 Quantifications of DAB and two-colour staining area from were performed with IHC image analysis

460 toolbox for ImageJ. Two full frame $4 x$ pictures adjusted for white background from each patient spinal 461 cord tissue were analyzed to get per-patient average $\mathrm{n}$ result. Quantification for immunofluorescence 462 in mouse spinal cords were performed by setting a common pixel intensity threshold and calculating 463 pixel-intensity within positive area using ImageJ. A typical 3-4 frames taken at 20x objective were used 
to obtain per mouse average intensity. Pictures are presented as 3D renderings of $16 \mu \mathrm{m}$ thick sections from $10 \mathrm{z}$ stacks and presented on white background using open source Icy software ver. 1.9.9.1. Perivascular space area in electron microscopy images was calculated from 3-5 regions of interest within the ventral horn grey matter that contained blood vessels acquired once from a group of 4 mice per genotype. First, we measured the areas outlined by mural and astrocyte basement membrane (BM). The gap was then calculated by subtracting the mural from astrocyte BM areas and divided by the mural BM area to normalize for individual vessel diameter.

\section{Statistics and reproducibility}

473 For immunostaining and transmission electron microscopy quantifications blinding was performed by 474 third party concealment of treatments or genotypes and assignment of numeric codes to each group.

475 Experiments were performed twice unless indicated otherwise. Representative graphs such as 476 basement membrane structure presented by transmission electron microscopy or by histology 477 stainings were based on observations found in 4 out of 4 analyzed animals or human cases or 478 quantified in respective data panels. Data are expressed as median \pm SEM. Box plots represent median, 479 Statistical comparisons were made with GraphPad Prism or R analysis software using Student's or 480 Wilcoxon tests (2-tailed unless otherwise indicated). For all tests, a p-value of 0.05 or less was considered significant.

\section{Patient cohorts for proteomics plasma profiling.}

484 Netherlands (Utrecht). Plasma samples and patient selection from Utrecht UMC was performed as 485 described previously ${ }^{51}$. In short: patients diagnosed with suspected, possible, probable or definite ALS according to the El Escorial criteria were included. Patients with progressive muscular atrophy, primary lateral sclerosis and progressive bulbar palsy were excluded from analysis. In order to determine whether a patient fulfilled the El Escorial criteria, the correspondence of the neurologist, including results of neurophysiological examination, was scrutinized. The controls included plasma from general hospital admission from patients without motor neuron disease. Germany (UIm). Patients were diagnosed according to the El Escorial criteria including possible ALS. The controls were patients were sampled because of headache, differential diagnoses subarachnoid hemorrhage or differential diagnoses meningitis.

494 Belgium (Leuven). A total of 134 patients with ALS and 27 control patients were consecutively included in a prospective manner between April 2014 and September 2016. All patients were seen at the Neuromuscular Reference Center (NMRC) of the University Hospitals Leuven. The patients with ALS were diagnosed according to the Awaji and revised El Escorial criteria. Sampling occurred during the diagnostic phase of the patient with ALS. The control cohort consisted out of 20 neurologic non-motor neuron disease controls and 7 patients with nonspecific subjective complaints for whom an underlying neurologic condition was ruled out upon neurologic examination. 
Blood samples were obtained during the first visit to the NMRC. Serum was extracted after 10 minutes

502 of centrifugation at $1955 \mathrm{~g}$, transferred into coded cryovials of $1 \mathrm{~mL}$ and stored at $-80^{\circ} \mathrm{C}$.

503 Sweden (Stockholm). Recruited patients received a diagnosis of ALS the Karolinska ALS center, an

504 outpatient clinic that manages all ALS patients in the Greater Stockholm area. ALS patients who met

505 the revised El Escorial criteria for definite, probable and probable laboratory-supported ALS were

506 included..$^{52}$ Patients that emigrated from the Stockholm region during the study period were excluded.

507 ALS patients were regularly evaluated by a neurologist, who registered their clinical characteristics in

508 the Swedish Motor Neuron Disease Quality Register. ${ }^{53}$ We further included siblings and partners of the

509 ALS patients as healthy controls. We performed a neurological exam of the controls but did not assess

510 their health status further. Healthy controls were recruited shortly after the diagnosis of the index

511 patient (usually within six months). Of the included siblings, one sibling was related to an ALS patient

512 that carried a C9orf72 repeat expansion, and one sibling was related to an ALS patient with a SOD1

513 mutation. For ALS patients, CSF and blood were collected at the time of diagnosis (+/- 90 days). All

514 patients were offered repeated blood (once every three to six months) and CSF sampling (once per

515 year). CSF and blood were collected from the ALS mimics during the diagnostic work-up, and from the

516 healthy controls shortly after the diagnosis of the index patient. CSF was obtained through lumbar

517 puncture directly into polypropylene tubes. The CSF samples were centrifugated for ten minutes at

$518400 \mathrm{~g}$ at room temperature. Plasma was collected close to the date of the lumbar puncture, and

519 centrifugated for ten minute at 2,000 $\mathrm{g}$ at room temperature. Aliquots of $1 \mathrm{ml}$ (CSF) and 800

$520 \mu \mathrm{L}$ (plasma) were directly frozen and stored in $-80^{\circ} \mathrm{C}$. NFL content in CSF was measured using the

521 UmanDiagnostics ELISA kit, Sweden (cat no 10-7001).

522

\section{Affinity proteomics of plasma}

524 -Antibody selection

525 Protein targets for plasma analysis were chosen based on mRNA transcript specificity to perivascular

526 fibroblasts, their induction in the human or mouse transcriptomics and based on antibody availability

527 within the Human Protein Atlas (HPA) project. A total of 32 antigen-purified and protein microarray

528 validated antibodies were selected for 15 unique proteins. Results presented in the figures were based

529 on antibodies against COL6A1 (HPA019142), SPP1 (HPA005562 or HPA027541).

530

-Suspension bead array

532 The procedure for suspension bead arrays was performed as described previously ${ }^{54,55}$. In short,

533 samples were distributed in 96-well microtiter plates, diluted 1:10 in phosphate buffered saline, and

534 the protein content directly labeled with biotin. For the bead array, antibodies were immobilized onto

535 magnetic color-coded beads with one bead identity corresponding to a certain antibody. Samples were

536 then further diluted 1:50 in an assay buffer, heat treated at $56^{\circ} \mathrm{C}$ for $30 \mathrm{~min}$, combined into a 384-well

537 microtiter plate, and incubated with the bead array at RT on a shaker overnight. Unbound proteins 
were removed by washing and proteins on the beads were detected through a streptavidin-conjugated fluorophore (Invitrogen.com). Results from the FlexMap3D instrument (Luminex Corp.Com) were reported per bead identity as median fluorescence intensities (MFI). The CSF analysis was performed similar as for plasma with some minor adjustments as reported previously in (Pin et al 2019 (PMID 31432421). First, 15 ul of the samples were labelled with biotin in a protein containing buffer (PBS supplemented with $0.5 \%(\mathrm{w} / \mathrm{v})$ bovine serum albumin and $0.1 \%(\mathrm{w} / \mathrm{v})$ rabbit IgG at an end dilution of

$5441 / 2$. For the assay, the samples were diluted $1 / 8$ in assay buffer to and end volume of 50 ul before heat 545 treatment.

-Antibody validation

The SPP1 antibody was validated with Western blot. Plasma samples were diluted 1:40 in MilliQ and proteins separated on a gel before blotted on a membrane (all Invitrogen). Detection antibody was applied at $1 \mu \mathrm{g} / \mathrm{mL}$ and binding allowed at $4^{\circ} \mathrm{C}$ overnight followed by readout through a chemiluminescent substrate (BioRad.Com).

552

-Suspension bead array data processing and statistical analysis

Data were processed and visualized in $R$ (v. 3.6.1). Samples with less than 20 counted beads per identity as well as technically failed samples were excluded from further analysis. To diminish any plate effect, multidimensional normalization was applied with the assumption that the mean of each plate for each antibody should be close. MA-LOESS normalization was thereafter applied to reduce effects associated to the different assay plates followed by log transformation of the datasets. Coefficients of variation were calculated based on a pool of samples analyzed in triplicate in each 96-well plate. For combined cohort analysis the datasets were median scaled. Association between groups was assessed by Wilcoxon rank sum tests.

-Optimization of plasma value cut-offs

564 To consider the effect of elevated protein levels, we determined optimal cut points to divide cohorts in

565 "low" or "high" level groups for the proteins in question. Cut points were based on patient protein

566 fluorescence intensity values in respect to survival. We used maximally selected rank statistics with the

567 logrank test similar to Kaplan-Meier analysis using the "maxstat" package v.0.7-25 (https://CRAN.R-

568 project.org/package=maxstat). All patient protein values were tested and considered as cut points

569 excluding 10 percent from the upper and lower end of the protein values range. Survival was analyzed

570 using "A Package for Survival Analysis in S" version 2.38. https://CRAN.R-project.org/package=survival.

571 Kaplan-Meier graphs were plotted based on the empirical threshold groups using "survminer" package

572 v. 0.4 .4 (https://CRAN.R-project.org/package=survminer). The protein values for COL6A1 in the Belgian

573 and Swedish cohorts yielded no clear cut point for survival groups and results shown are based on the

574 threshold at the median value. 
In order to assess the risk of having elevated protein levels in comparison to other variables we created univariate and multivariate Cox proportional hazard models. Variables tested were disease onset type, gender and age at sampling. Both univariate and multivariate models were corrected for sampling delay and cohort effect whenever applicable. Sampling delay was added as a stratification term, whereas we used a cluster term on cohort identities to adjust for possible unknown correlations within cohorts. The latter being a generalized estimating equation (GEE) term. Above analyses were performed in R version 3.6.1 (https://www.R-project.org/). Patient plasma data and analysis scripts for cut-off optimization and Cox proportional hazard are available at:

\section{Analysis of ALSFRS data at the time of plasma/CSF sampling}

In this analysis, we aimed to assess the ALSFRS value at the time point of plasma/CSF sampling. Table 2 https://figshare.com/s/4c89e57b39620d020f8d displays the numbers of ALS patients who had ALSFRS data available in each of the cohorts, and who were included in the analysis. Patients were included if their date of clinical onset was known (at which point the ALSFRS $=48$ ) and they had at least one ALSFRS measurement available with known date. sampling or within \pm 10 days. For these patients, we used the actual ALSFRS measure. and used that formula to estimate ALSFRS at the plasma/CSF sampling time point. When we had three or more ALSFRS data points, we used a sigmoid curve, following a method recently published ${ }^{56}$. This study has determined that when patients are followed longitudinally over multiple time points, the ALSFRS data follows a sigmoidal curve, being characterized by an initial phase of relatively gradual decline near the initial point of disease onset, followed by a time interval of relatively fast decline, and leading to a final stage of slower decline when patients are at a severe stage. The sigmoid function allows describing the whole ALSFRS temporal progression using two parameters, D50 and dx, as expressed by the following formula ${ }^{56}$ :

607

$$
y=\frac{48}{1+e^{\frac{(x-D 50)}{d x}}}
$$


612 numerator $=$ corresponds to the ALSFRS score (48) assumed the time of clinical onset

$613 \mathrm{D} 50=$ parameter that is equal to the number of months after clinical onset $(\mathrm{x})$ when the ALSFRS

614 score (y) drops to half of its value at clinical onset, i.e., when y drops to 24

$615 \mathrm{dx}=$ slope of the sigmoid curve at $\mathrm{x}=\mathrm{D} 50$, with low $\mathrm{dx}$ values corresponding to steeper decline,

616 and high $\mathrm{dx}$ values corresponding to slower decline at $\mathrm{x}=\mathrm{D} 50$

618 For each individual patient, the ALSFRS-R scores were fitted to the sigmoid function using Microsoft ${ }^{\circledR}$

619 Excel Add-In Solver tool, by an iterative procedure used to simultaneously estimate the D50 and dx

620 values that provide the best fit for the longitudinal ALSFRS-R data. The sigmoid model was applied if

621 there were at least 3 time points for each patient.

622

\section{Data availability}

624 Human ALS patient transcriptome datasets and analysis scripts are available at:

625 https://github.com/NathanSkene/ALS Human EWCE

$626 S O D 1^{G 93 A}$ mouse transcriptome datasets and analysis scripts are available at:

627 https://github.com/NathanSkene/ALS Mouse EWCE

$628 T A R D B P^{0331 K / 0331 K}$ mouse transcriptome datasets and analysis scripts are available at:

629 https://github.com/szczepinskaa/ALS TDP-43.git

630 Images and analysis scripts for human histology are deposited and available under the following links:

631 SPP1 in Red - https://figshare.com/s/3ad17913ca4fb2e99b80

632 COL6A1 in Red - https://figshare.com/s/f318c332f4fbc31b48a0

633 SPP1 in DAB - https://figshare.com/s/ee294c7715f77db7df14

634 COL6A1 in DAB - https://figshare.com/s/8c0ecbe9b3fbc63cfefo

635 Additional data tables are available at: https://figshare.com/s/4c89e57b39620d020f8d

636 Patient plasma data and analysis scripts for cut-off optimization and Cox proportional hazard are

637 available at: https://github.com/lewandowskilab/PVF Manuscript

\section{Code availability}

640 Human ALS patient transcriptome analysis scripts are available at:

641 https://github.com/NathanSkene/ALS Human EWCE

$642 S O D 1^{G 93 A}$ mouse transcriptome analysis scripts are available at:

643 https://github.com/NathanSkene/ALS Mouse EWCE

$644 T A R D B P^{0331 K / 0331 K}$ mouse transcriptome analysis scripts are available at:

645 https://github.com/szczepinskaa/ALS TDP-43.git

646 Images and analysis scripts for human histology are deposited and available under the following links: 
SPP1 in Red training model - https://figshare.com/s/3ad17913ca4fb2e99b80

648 COL6A1 in Red training model - https://figshare.com/s/f318c332f4fbc31b48a0

649 SPP1 in DAB training model - https://figshare.com/s/ee294c7715f77db7df14

650 COL6A1 in DAB training model and macro - https://figshare.com/s/8c0ecbe9b3fbc63cfef0

651 Patient plasma analysis scripts for cut-off optimization and Cox proportional hazard are available at:

652 https://github.com/lewandowskilab/PVF Manuscript

653

654

655

656

657

\section{Extended data figures}

659 (see separate document)

660

\section{Acknowledgements}

662 S.A.L. is supported by the Olle Engkvist Byggmästare Foundation (SLS-499431), Ulla-Carin Lindquists

663 stiftelse för ALS-forskning, Åhléns foundation (mA2/h17, 203074), Thierry Latran Foundation (FIB-ALS)

664 and Neuroförbundet. N.S. was supported by Wellcome Trust (108726/Z/15/Z), Edmond J. Safra

665 Foundation, Lily Safra and UK Dementia Research Institute. We thank the ALS Stichting grant "The

666 Dutch ALS Tissue Bank" (E.A.) and Netherlands Brain Bank (E.H.) for providing the ALS tissue samples.

667 We acknowledge the team who helped in the collection of ALS tissue samples (Prof. dr. D. Troost,

668 Prof. dr. M. de Visser, Dr. A.J. van der Kooi and Dr. J. Raaphorst). U.K. and C.I. are supported by

669 Björklunds Fund, the Ulla-Carin Lindquist Foundation, Neuro Sweden, SLL Halsa Medicin och Teknik.

670 E.R-V. is supported by Swedish Alzheimer Foundation (Alzheimerfonden), Swedish Dementia

671 Association (Demensfonden), Gun \& Bertil Stohne's Foundation, and Gamla Tjänarinnor Foundation.

672 This project has received funding from the European Research Council (ERC) under the European

673 Union's Horizon 2020 research and innovation program (grant agreement $n^{\circ} 772376-$ EScORIAL

674 awarded to J.V. R.A.H. is supported by Alltid Litt Sterkere, AlzheimerFonden, Swedish Medical

675 Research Council, Swedish brain foundation and Karolinska Institutet. M.L. was supported by a grant

676 from the Knut and Alice Wallenberg Foundation (2012.0091). This study was also supported by grants

677 to P.N. from the Swedish FTD initiative funded by the Schörling Family Foundation and the KTH Center

678 for Applied Precision Medicine (KCAP) funded by the Erling-Persson Family Foundation. P.V.D. holds a

679 senior clinical investigatorship of FWO-Vlaanderen and is supported by E. von Behring Chair for

680 Neuromuscular and Neurodegenerative Disorders, the ALS Liga België and the KU Leuven funds "Een

681 Hart voor ALS", "Laeversfonds voor ALS Onderzoek" and the "Valéry Perrier Race against ALS Fund".

682 Several authors of this publication are member of the European Reference Network for Rare

683 Neuromuscular Diseases (ERN-NMD). We would also like to thank Kjell Hultenby at Karolinska 

Marklund for assistance with selection and harvest of $S O D 1^{G 93 A}$ mouse tissues. S.A.L would like to thank the unpaid interns on Erasmus scholarships for their efforts and contributions.

\section{Author contributions}

N.S. and S.A.L. designed transcriptomics enrichment experiments.

N.S. and A.S. performed the computational analysis of enrichment experiments. M.T., I.S.A., A.D. and P.L. performed additional transcriptomics analysis. M.L., J.D.G. and S.A.L. facilitated and performed mouse histology and immunostaining. I.V.G.A. performed histology validation experiments. L.E., M.T. and S.A.L. performed electron microscopy imaging and quantifications. R.A.H., E.H., J.A., C.M. and E.A., facilitated and performed human histology staining. Clinical centers in: Ulm (A.H., A.L.), Utrecht (H.V.B., J.V.), Leuven (J.D.V., M.D.S., K.P., P.V.D.) and Stockholm (C.I., U.K.) - designed patient and control cohorts and provided plasma or CSF samples. S.A.L., A.M. and J.R. designed and chose protein targets for plasma profiling. associations, and clinical parameter statistics. S.A.L. wrote the manuscript with input from the co-authors. P.N., and S.A.L. oversaw all aspects of the study.

Competing Interests. We declare that the authors do not have competing interests.

Figure 1. ALS patients show increased transcriptional activity of perivascular fibroblast cell gene markers which occur at presymptomatic disease stage in SOD $1^{G 93 A}$ and TARDBP ${ }^{Q 331 K}$ mice. (A) Schematic of expression weighted cell type enrichment (EWCE) analysis. Cell type-specific gene rankings from single cell sequencing data allow to infer cell type activity in bulk tissue transcriptomes.

714 (B) Enrichment $z$ - scores for up and down-regulated genes in ten cell type classes in sALS patients, $715 S O D 1^{G 93 A}$ and TARDBP $P^{Q 331 K}$ mice. In the $S O D 1^{G 93 A}$ mice, onset of neuromuscular junction decoupling (8 716 weeks) and clinical symptoms (peak body weight - 16 weeks) are indicated with arrowheads. P-values

717 are specified in Ext. data Fig. 2. (C) Cell type RNA specificity for genes enriched in perivascular

718 fibroblasts. $\mathrm{N}$ number of cells per category is described in Ext. data Fig. 1. Bars show median count of 719 RNA per cell \pm SEM. (D) Perivascular fibroblast specific gene activity in sALS patient spinal cords. SALS $\mathrm{n}=12, \mathrm{Ctrl} n=8$. Boxplots show median, $2 \mathrm{nd}-3 \mathrm{rd}$ quartile and whiskers show $+/-1.5$ of the IQR. Two- 
tailed t-test $p$-values are indicated next to brackets. (E) Expression of perivascular fibroblast enriched genes in $S O D 1^{G 93 A}$ ( $p$-value for ANOVA in early (4-10 weeks) and late (14-18 weeks) timepoints) and TARDBP $P^{0331 K}$ mice ( $p$-values for two-tailed t-test). (F) Perivascular fibroblasts have distinct mRNA expression markers (median RNA molecule count with \pm SEM). (G) Schematic illustration represents reported PVF location between astrocytes and mural/endothelial cells. (H) Transmission electron microscopy of mouse spinal cord tissue points to location of perivascular fibroblasts cells (PVF - shaded red) between basement membrane layers that delineate astrocyte endfeet (AC - blue) and mural cells (MC - brown), (EC - yellow: endothelial cell, aBM - astrocyte basement membrane, mBM - mural basement membrane). Scale bar $2 \mu \mathrm{m}$.

Figure 2. Perivascular fibroblast marker proteins COL6A1 and SPP1 accumulate in enlarged perivascular spaces during ALS progression. (A) Col6a1 and Spp1 mRNA specificity within CNS cell types. Bars represent relative count of RNA per cell \pm SEM. (B) COL6A1 and SPP1 histochemistry in SALS and control spinal cords, bar: $10 \mu \mathrm{m}$. (C) Quantifications of human tissue histochemistry from full frame $4 x$ photos. SALS and CtrI $n=4$ individuals (2-tailed t-test $p$-value). All boxplots show median, $2 n d-3 r d$ quartile and whiskers show +/-1.5 of the IQR. (D) Col6a1 and Spp1 accumulate around blood vessels (outlined with podocalyxin - cyan) in 14 week SOD $1^{G 93 A}$ mouse spinal cords. Immunofluorescence zstack renderings of $16 \mu \mathrm{m}$ thick sections, bars: $100 \mu \mathrm{m}$ (overview), 10 $\mu \mathrm{m}$ (insert). (E) Quantifications of immunofluorescence stainings in mice from full-frame $20 x$ photos. SOD $1^{G 93 A}$ and $B L / 6 n=4, S O D 1^{w t} n=3$ mice (2-tailed t-test p-value). (F) Increased perivascular spaces appear in presymptomatic (8 weeks) $S O D 1^{G 93 A}$ mice spinal cords. Immunofluorescence for vascular (Col4a1) and astrocyte (Lama1) basement membranes, bar: $10 \mu \mathrm{m}$. (G) Electron microscopy (tEM) of increased perivascular spaces in 14 week SOD $1^{G 93 A}$ mice. Astrocyte (red) and vascular (blue) basement membranes are indicated with lines. Perivascular space is indicated with asterisk, bar: $5 \mu \mathrm{m}$. (H) COL6A1 and SPP1 accumulate within increased perivascular spaces (outlined with COL4A1) in spinal cords of SALS patients. 2 color

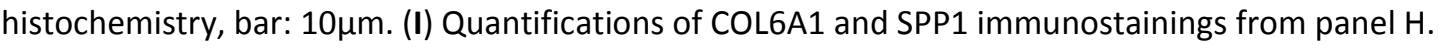
sALS and Ctrl $n=4$ individuals (two-tailed t-test $p$-value). (J) Quantifications of perivascular space increase in $S O D 1^{G 93 A}$ mice from panel G $\left(S O D 1^{G 93 A}\right.$ and $\mathrm{BL} / 6 \mathrm{n}=4$ mice $)$ and in SALS patients from panel $\mathrm{H}$ (sALS and Ctrl $\mathrm{n}=4$ individuals) (two-tailed t-test $\mathrm{p}$-value). (K) Schematic representation of perivascular fibroblast activity and enlarged perivascular spaces in ALS. protein in plasma as measured by the HPA027541 antibody. Threshold selection and Kaplan-Meier survival estimates of ALS patients in discovery cohorts (Netherlands, Germany and Belgium, $n=452$ ) and in the replication cohort (Sweden, $n=122$ ). Red color indicates thresholded protein level. Thresholds are established using maximally selected log rank statistics. Boxplots show median, 2nd-3rd 
758 patients within each arm and Kaplan-Meier logrank p-values. (B-C) Uni- and multivariate Cox

759 proportional hazard models for continuous increase of plasma SPP1 relative to hazard ratios indicated

760 by bulbar onset type, neurofilament light (NFL) in CSF (in the replication cohort), gender and plasma

761 sampling age. Whiskers represent $95 \% \mathrm{Cl}$. Cohort identity was additionally used as covariate in

762 multivariate models.

763 
765 1. Cook, C. \& Petrucelli, L. Genetic Convergence Brings Clarity to the Enigmatic Red Line in ALS. Neuron 101, 1057-1069 (2019).

2. Spiller, K. J. et al. Microglia-mediated recovery from ALS-relevant motor neuron degeneration in a mouse model of TDP-43 proteinopathy. Nat. Neurosci. 21, 329-340 (2018).

3. Boillée, S. et al. Onset and progression in inherited ALS determined by motor neurons and

4. Lewandowski, S. A. et al. Presymptomatic activation of the PDGF-CC pathway accelerates onset

5. Brown, R. H. \& Al-Chalabi, A. Amyotrophic Lateral Sclerosis. N. Engl. J. Med. 377, 162-172 (2017).

6. Al-Chalabi, A. et al. Analysis of amyotrophic lateral sclerosis as a multistep process: A population-based modelling study. Lancet Neurol. 13, 1108-1113 (2014).

7. Rosen, D. R. et al. Mutations in $\mathrm{Cu} / \mathrm{Zn}$ superoxide dismutase gene are associated with familial amyotrophic lateral sclerosis. Nature 362, 59-62 (1993).

8. Sreedharan, J. et al. TDP-43 mutations in familial and sporadic amyotrophic lateral sclerosis. Science 319, 1668-72 (2008).

9. $\quad \mathrm{Wu}, \mathrm{C} . \mathrm{-H}$. et al. Mutations in the profilin 1 gene cause familial amyotrophic lateral sclerosis. Nature 488, 499-503 (2012).

10. Kang, S. H. et al. Degeneration and impaired regeneration of gray matter oligodendrocytes in amyotrophic lateral sclerosis. Nat. Neurosci. 16, 571-9 (2013).

11. Zhong, Z. et al. ALS-causing SOD1 mutants generate vascular changes prior to motor neuron degeneration. Nat. Neurosci. 11, 420-422 (2008).

12. Zeisel, A. et al. Cell types in the mouse cortex and hippocampus revealed by single-cell RNAseq. Science (80-. ). 347, 1138-42 (2015).

13. Marques, S. et al. Oligodendrocyte heterogeneity in the mouse juvenile and adult central nervous system. Science (80-. ). 352, 1326-1329 (2016).

14. Zeisel, A. et al. Molecular Architecture of the Mouse Nervous System. Cell 174, 999-1014.e22 (2018).

15. Skene, N. G. \& Grant, S. G. N. Identification of vulnerable cell types in major brain disorders using single cell transcriptomes and expression weighted cell type enrichment. Front. Neurosci. 10, 1-11 (2016).

16. Clark, J. A., Southam, K. A., Blizzard, C. A., King, A. E. \& Dickson, T. C. Axonal degeneration, distal collateral branching and neuromuscular junction architecture alterations occur prior to symptom onset in the SOD1G93A mouse model of amyotrophic lateral sclerosis. J. Chem. Neuroanat. 76, 35-47 (2016).

17. Hall, E. D., Oostveen, J. A. \& Gurney, M. E. Relationship of microglial and astrocytic activation to 
disease onset and progression in a transgenic model of familial ALS. Glia 23, 249-56 (1998).

802

803

804

805

806

807

808

809

810

811

812

813

814

815

816

817

818

819

820

821

822

823

824

825

826

827

828

829

830

831

832

833

834

835

836

837

18. Weydt, P., Hong, S. Y., Kliot, M. \& Möller, T. Assessing disease onset and progression in the SOD1 mouse model of ALS. Neuroreport 14, 1051-4 (2003).

19. White, M. A. et al. TDP-43 gains function due to perturbed autoregulation in a Tardbp knock-in mouse model of ALS-FTD. Nat. Neurosci. 21, 552-563 (2018).

20. Chiu, I. M. et al. A Neurodegeneration-Specific Gene-Expression Signature of Acutely Isolated Microglia from an Amyotrophic Lateral Sclerosis Mouse Model. Cell Rep. 4, 385-401 (2013).

21. Sun, S. et al. Translational profiling identifies a cascade of damage initiated in motor neurons and spreading to glia in mutant SOD1-mediated ALS. Proc. Natl. Acad. Sci. U. S. A. 112, E69937002 (2015).

22. Vanlandewijck, M. et al. A molecular atlas of cell types and zonation in the brain vasculature. Nature 554, 475-480 (2018).

23. Ma, Q., Ineichen, B. V., Detmar, M. \& Proulx, S. T. Outflow of cerebrospinal fluid is predominantly through lymphatic vessels and is reduced in aged mice. Nat. Commun. 8, (2017).

24. Kapoor, K. G., Katz, S. E., Grzybowski, D. M. \& Lubow, M. Cerebrospinal fluid outflow: An evolving perspective. Brain Res. Bull. 77, 327-334 (2008).

25. Tam, O. H. et al. Postmortem Cortex Samples Identify Distinct Molecular Subtypes of ALS: Retrotransposon Activation, Oxidative Stress, and Activated Glia. Cell Rep. 29, 1164-1177.e5 (2019).

26. Westeneng, H.-J. et al. Prognosis for patients with amyotrophic lateral sclerosis: development and validation of a personalised prediction model. Lancet Neurol. 17, 423-433 (2018).

27. Braak, H. et al. Amyotrophic lateral sclerosis-a model of corticofugal axonal spread. Nat. Publ. Gr. 9, 708-714 (2013).

28. Chiu, A. Y. et al. Age-Dependent Penetrance of Disease in a Transgenic Mouse Model of Familial Amyotrophic Lateral Sclerosis. Mol. Cell. Neurosci. 6, 349-362 (1995).

29. Gille, B. et al. Inflammatory markers in cerebrospinal fluid : independent prognostic biomarkers in amyotrophic lateral sclerosis ? 1-9 (2019). doi:10.1136/jnnp-2018-319586

30. Turner, M. R., Goldacre, R., Talbot, K. \& Goldacre, M. J. Cerebrovascular injury as a risk factor for amyotrophic lateral sclerosis: Table 1. J. Neurol. Neurosurg. Psychiatry 87, 244-246 (2016).

31. Garton, F. C., Trabjerg, B. B., Wray, N. R. \& Agerbo, E. Cardiovascular disease, psychiatric diagnosis and sex differences in the multistep hypothesis of amyotrophic lateral sclerosis. Eur. J. Neurol. 1-10 (2020). doi:10.1111/ene.14554

32. Rule, R. R., Schuff, N., Miller, R. G. \& Weiner, M. W. Gray matter perfusion correlates with disease severity in ALS. Neurology 74, 821-827 (2010).

33. Murphy, M. J. et al. Widespread cerebral haemodynamics disturbances occur early in amyotrophic lateral sclerosis. Amyotroph. Lateral Scler. 13, 202-9 (2012).

34. Van Laere, K. et al. Value of 18fluorodeoxyglucose-positron-emission tomography in 
amyotrophic lateral sclerosis: a prospective study. JAMA Neurol. 71, 553-61 (2014).

35. Wolak, T. Osteopontin - a multi-modal marker and mediator in atherosclerotic vascular disease. Atherosclerosis 236, 327-37 (2014).

36. Chiocchetti, A. et al. Osteopontin Bridging Innate and Adaptive Immunity in Autoimmune Diseases. J. Immunol. Res. 2016, 1-15 (2016).

37. Zhao, H. et al. The role of osteopontin in the progression of solid organ tumour. Cell Death Dis.

40. Brown, R. et al. Understanding the role of the perivascular space in cerebral small vessel

41. Skene, N. G. \& Grant, S. G. N. Identification of vulnerable cell types in major brain disorders

42. Lerman, B. J. et al. Deletion of galectin-3 exacerbates microglial activation and accelerates

43. Gautier, L., Cope, L., Bolstad, B. M. \& Irizarry, R. A. affy--analysis of Affymetrix GeneChip data at

44. Durinck, S., Spellman, P. T., Birney, E. \& Huber, W. Mapping identifiers for the integration of

45. Ritchie, M. E. et al. limma powers differential expression analyses for RNA-sequencing and

46. Rabin, S. J. et al. Sporadic ALS has compartment-specific aberrant exon splicing and altered cell-

47. Harbor, B. Working with ALS Mice. Jax 1-28 (2009).

48. Ludolph, A. et al. A revision of the El Escorial criteria - 2015. Amyotroph. Lateral Scler. Front.

49. van Vliet, E. A. et al. Expression and Cellular Distribution of P-Glycoprotein and Breast Cancer Resistance Protein in Amyotrophic Lateral Sclerosis Patients. J. Neuropathol. Exp. Neurol. 79, 266-276 (2020).

50. Casula, M. et al. Toll-like receptor signaling in amyotrophic lateral sclerosis spinal cord tissue. Neuroscience 179, 233-243 (2011).

1. Huisman, M. H. B. et al. Population based epidemiology of amyotrophic lateral sclerosis using capture-recapture methodology. J. Neurol. Neurosurg. \&amp;amp; Psychiatry 82, 1165 LP-1170 
(2011).

876 52. Brooks, B. R., Miller, R. G., Swash, M. \& Munsat, T. L. El Escorial revisited: Revised criteria for the diagnosis of amyotrophic lateral sclerosis. Amyotroph. Lateral Scler. Other Mot. Neuron

878 Disord. 1, 293-299 (2000).

879

53. Wallin, N. et al. The Swedish motor neuron disease quality registry. (2018).

880 doi:10.1080/21678421.2018.1497065

881 54. Schwenk, J. M., Gry, M., Rimini, R., Uhlén, M. \& Nilsson, P. Antibody Suspension Bead Arrays $882 \quad$ within Serum Proteomics. J. Proteome Res. 7, 3168-3179 (2008).

883 55. Häggmark, A. et al. Plasma profiling reveals three proteins associated to amyotrophic lateral

884 sclerosis. Ann. Clin. Transl. Neurol. 1, 544-53 (2014).

885 56. Poesen, K. et al. Neurofilament markers for ALS correlate with extent of upper and lower motor 886 neuron disease. Neurology 88, 2302-2309 (2017).

887

888 